\title{
Public Health and Family Medicine: An Opportunity
}

\author{
Doug Campos-Outcalt, MD, MPA
}

The current national concern about bioterrorism and the inadequacy of the public health infrastructure offers an opportunity for American family physicians to assume a key role in the front line of the US public health system. To meet this challenge, family medicine educators need to insure that all family physicians are well trained to collaborate effectively with their local and state public health departments in fulfilling pertinent public health functions under routine and emergency circumstances.

Public health and family medicine are natural allies. Both can be more effective and achieve more when they work collaboratively. However, collaboration often does not occur. Medicine and public health have evolved separately, are usually taught in separate colleges, and are practiced in different settings and systems with different incentives and goals. Public health and medicine have different values, philosophies, and cultures.

Family medicine has attempted to bridge this gap by integrating community-oriented primary care (COPC) into undergraduate and residency training programs, ${ }^{1,2}$ but there has been little effect on the eventual practice of most community family physicians. ${ }^{3}$ Several of the processes of COPCdefining and characterizing the community, describing community health problems, and implementing programs to affect them-are functions that many local health departments are better equipped, motivated, and rewarded to fulfill than are physicians, who are usually quite occupied keeping up with the demands of individual patients. COPC would be better implemented through a public health/community physician collaboration,

Submitted, revised, 12 February 2004.

From the Maricopa County Department of Public Health, Phoenix, Arizona, and the Department of Family and Community Medicine, University of Arizona College of Medicine, Phoenix. Address correspondence to: Doug Campos-Outcault, MD, MPA, Department of Family and Community Medicine, University of Arizona College of Medicine, 4001 North 3rd Street, Suite 415, Phoenix, AZ 85012-2085 (e-mail: dougco@u.arizona.edu). with each fulfilling distinct and important functions.

In this article, I suggest ways we can begin to bridge this public health/medicine separation. I first suggest a definition for public health that includes not only what public health is and does but also what it is not. I then delineate roles for family physicians in the public health system and conclude with a recommended set of skills and knowledge for 4 levels of expertise, along with training options for achieving them.

\section{What Is Public Health?}

Public health is the discipline that promotes and protects the health of the entire public. The Core Public Health Functions Steering Committee, established by the US Public Health Service, constructed a list of 10 essential public health functions, shown in Table $1 .^{4}$ These 10 functions allow public health to prevent epidemics and the spread of disease; protect against environmental hazards; prevent injuries; promote healthy behaviors; respond to disasters and assist in recovery from disasters; and assure the quality and accessibility of health services. The Institute of Medicine Work Group on the Future of Public Health condensed these functions into 3 broader areas of responsibility: assessment, policy development, and assurance. $^{5}$

Public health is not, and should not be equated with, publicly funded safety-net health care. Although safety-net health care is an important function and contributes to better overall public health, providing medical services to those who lack access to other sources of care is a medical system function, based on a medical model of individual care. Public health is population based; the patient is the entire community.

The basic science and fundamental tool of public health is epidemiology. The classic public health approach to a problem is to use epidemiology to study and define it, find the cause, intervene to address the cause, and evaluate to determine 
- Monitor health status to identify community health problems.

- Diagnose and investigate health problems and health hazards in the community.

- Enforce laws and regulations that protect health and ensure safety.

- Inform, educate, and empower people about health issues.

- Mobilize community partnerships to identify and solve health problems.

- Link people to needed personal health services and assure the provision of health care when it is otherwise unavailable.

- Evaluate effectiveness, accessibility, and quality of personal and population-based health services.

- Assure a competent public health and personal health care workforce.

- Develop policies and plans that support individual and community health efforts.

- Research for new insights and innovative solutions to health problems.

whether the intervention works and the problem decreases. Public health interventions available include laws, regulations, product design, immunizations, antibiotics, taxes, and education, to name a few. This list makes some uncomfortable. It implies governmental involvement and interference in personal freedoms. The reality is that many public health activities do involve limiting individual choices. The factor that often determines the limits of public health achievement is how much interference with individual freedom the public is willing to accept to improve the health and protection of the whole community.

Although education to affect individual and community choices is an important tool, history has shown that, in many instances, the more individual choice can be eliminated from the equation, the better the outcome will be. This is contrary to our individualistic American values. It also seems to conflict with how family physicians interact with patients, by providing individualized education and counseling in an attempt to convince them to live healthier lives. However, individualized health education and community-wide public health interventions are actually complementary; both are important in achieving maximum public health.

The CDC published a series of articles on major public health accomplishments of the 20th century. ${ }^{6}$ The list includes what some experts consider the most important interventions in improving the public's health: immunizations, control of infectious disease (which has been a result of sanitation, clean water, and improved living conditions), motor vehicle safety, consumer product safety, food safety and improved nutrition, fluoridation of water, cardiovascular disease reduction, tobacco control, and achieving healthier mothers and babies. A close look at the accomplishments on this list will show that very few depended on individuals making optimal choices.

There are now many local and federal restrictions against smoking in pubic places. Most people live in areas where we do not allow the building or selling of houses without sanitation and clean water. We do not allow parents to decide if their children should be immunized before attending public school. (This is actually more complex to explore than space allows, but the thrust behind school immunization laws, despite individual optout options, is to compel immunization.) We do not allow new cars to be sold that do not meet safety standards. In none of these instances do we allow individual choices, in the hope that education will encourage healthy decisions. On the contrary, we depend on community acceptance of restrictions of certain freedoms and choices with the understanding that the overall good is thereby served. Local physicians have a vital role in influencing the community's acceptance of such public health interventions.

One of the newer challenges facing public health today is chronic disease. Much of the burden of chronic diseases is caused by individual lifestyle choices. The public is willing to accept only limited government interference with these choices because, except for the societal impact of medical costs and other adverse economic effects of disease, these lifestyle decisions seem to affect only the person making them. The only tool left to us is education, which can still achieve significant benefits. This is a major opportunity for family physicians, who are often the main source of information and education for individual patients and families. 


\section{The Role of Family Physicians in Public Health}

There are 5 functions that all family physicians should fulfill in their role as a vital component of the public health system: (1) implementing recommended preventive services guidelines in their practices; (2) serving as the front line of the surveillance system; (3) appropriately referring to the public health department; (4) accepting referrals from the public health department; and (5) interacting constructively with the local health department.

Family physicians perform many preventive services in their daily clinical practices, such as cancer screening, immunizations, and smoking cessation counseling. Keeping current on recommendations can be challenging but is made easier by guidelines promulgated the United States Preventive Task Force and the American Academy of Family Physicians. All states require reporting of specified infectious diseases. Although the usefulness of this practice is not always readily apparent to the practicing physician, these reports are important in detecting disease trends and outbreaks. The early detection of any future bioterrorist events will probably be a result of reporting by front-line clinicians.

Clinical and programmatic capabilities of local health departments vary considerably. Where the local health department runs clinics for tuberculosis and sexually transmitted diseases, family physicians can refer their patients to these services when needed (for instance, when a dark-field microscopic examination is needed to diagnose syphilis). In some instances, particularly in rural areas, the health department may contract these services to local physicians who must have up-to-date knowledge of diagnosis and treatment guidelines. Other public health services that can be of use to family physicians and their patients include women, infants, and children nutrition programs, sexually transmitted disease contact tracing, directly observed therapy for tuberculosis, and laboratory services for diseases of public health importance.

Interacting constructively with the local health department consists of correctly treating tuberculosis, sexually transmitted diseases, and other diseases of public health significance; recognizing diseases, or the potential for diseases, of public health importance (eg, knowing rash and fever might be measles or rubella); implementing office infection control measures to help prevent spread of disease in the community; providing accurate information to patients and to the public that is consistent with the information being provided by public health authorities; and making constructive public remarks about the public health department. This does not mean that public health policies should not be openly discussed and debated; they should be. There are often controversies and disagreements that need to be resolved. This is best accomplished, however, in an atmosphere of mutual respect.

Many family physicians may have more involved public health roles, including serving as a sentinel practice, being a medical director of a clinic or health plan, serving on a local board of health, holding public office, or being a public health official. To prepare family physicians for these various roles with their local health departments, I suggest 4 levels of public health expertise: basic, intermediate, advanced, and leadership. All primary care physicians need basic expertise. Intermediate expertise would be for sentinel clinicians and health plan medical directors. Advanced training would be for those serving as consultants or communitybased medical directors to public health departments and on boards of health. Those aspiring to public health clinical and administrative leadership need additional skills. The knowledge and skills needed at each level are listed in Table 2.

\section{Training Methods}

The basic level skills and knowledge should be taught in the preclinical years of medical school as part of a course on preclinical medicine, behavioral science, community medicine, or public health, and reinforced in the required clerkships using both a didactic and experiential format. A fourth-year public health practice elective could then focus on intermediate level skills and knowledge. ${ }^{7}$

The family practice residency curriculum should review and insure the mastery of the basic level and incorporate the intermediate and advanced levels. This could be accomplished by including the content in didactics, insuring that the family practice clinic and faculty model these practices, and by developing an enhanced community medicine experience that includes these topics.

Advanced level training can be achieved in several ways. Family physicians can obtain an MPH 
I. Basic

Knowledge

4 Functions and powers of local health departments

4 How to interact effectively with state and local health departments

4 Roles of local, state and federal agencies with public health functions

- Medical care of sexually transmitted diseases, tuberculosis, other diseases important to public health

4 Evidence-based prevention recommendations

Appropriate use of public health resources (contact tracing, directly observed therapy, isolation and quarantine)

$\checkmark$ Role in emergencies and disasters

Skills

4 Reporting of reportable diseases and conditions

Accurate recording of vital statistics

4 Implementation of clinical prevention guidelines (screening, immunization, counseling, chemoprophylaxis, travel advice)

4 Interpretation of medical literature (basic epidemiology and statistics)

4 Recognition of unusual diseases and patterns

4 Finding information on environmental concerns

Referral to community resources

4 Counseling to achieve lifestyle change

II. Intermediate

All of the basic knowledge and skills plus:

Knowledge

4 More advanced epidemiology and statistics

4 What confirmatory labs to order to confirm important infectious diseases

4 Basics of environmental exposure risk assessment

Medical aspects of public health emergencies including bioterrorism

Skills

$\checkmark$ Recognition and treatment of unusual presentations and complications of sexually transmitted diseases and tuberculosis

Implementing clinical systems for increasing adherence to prevention guidelines

4 Designing an observational or interventional study

Diagnosis and treatment of infections caused by level A bioterrorism agents

III. Advanced

All of the basic and intermediate knowledge and skills plus:

Knowledge

4 Public health legal authority and police powers

- Medical aspects of disasters and other public health emergencies

$\checkmark$ Emergency medical transport systems

4 Medical examiner functions

Animal and vector control activities

4 Water system safety

4 Food safety

4 Medical information systems

4 Community social service and medical resources

Skills

4 Performing environmental risk assessments

4 Collection and transport of lab specimens

4 Designing medical information systems

4 Implementing quality improvement systems

- Using epidemiology and statistics for community health assessment

4 Communicating with the public and press

IV. Leadership

All of basic, intermediate, and advanced knowledge and skills plus:

Knowledge

4 Public policy formulation

4 Personnel systems

Budgeting and finance

4 Procurement procedures

4 Grants and contracts

4 Planning and evaluation

Skills

Legal responsibilities and limits

4 Developing a program and department budget

4 Developing and defending a policy initiative

4 Communicating with elected officials

- Developing collaborative relationships with public and private sector agencies

Managing personnel, facilities, and budgets

degree while in medical school, as part of a MDMPH program, while in residency, ${ }^{8}$ or after completion of residency. Another option is to complete a joint family practice-preventive medicine residency; there are now at least 7 of these joint pro- grams. ${ }^{9}$ Preventive medicine residency consists of 3 years of training: an internship or clinical year, a master's degree in public health, and an applied practicum year. Combining family practice and preventive medicine training can reduce the 
amount of time required for dual board eligibility from 6 years to 4 or 5 years.

The leadership of family medicine could help assure that learning is achieved through the advanced level by incorporating public health objectives into the core residency requirements. Family medicine educators who want to make leadership training available should explore creating more joint family practice-MPH and family practicepreventive medicine programs. The American Academy of Family Physicians could collaborate with the American College of Preventive Medicine and the Association of Teachers of Preventive Medicine to develop public health continuing education courses and certification programs for practicing physicians.

\section{Conclusion}

Public health and primary care are natural allies in the struggle to improve health. For the fields to work productively and collaboratively, each must understand what the other does; they must function as a team. Family physicians can lead the way for all medicine by providing training for all its residency graduates and practitioners on how to interact effectively with local health departments and how to assume positions of leadership in the public health infrastructure either as a public health official or as a community-based consultant and collaborator.

\section{References}

1. Longlett SK, Kruse JE, Wesley RM. Communityoriented primary care: critical assessment and implications for resident education. J Am Board Fam Pract 2001;14:141-7.

2. Plescia M, Konen JC, Lincourt A. The state of community medicine training in family practice residency programs. Fam Med 2002;34:177-82.

3. Longlett SK, Kruse JE, Wesley RM. Communityoriented primary care: historical perspective. J Am Board Fam Pract 2001;14:54-63.

4. Public Health Functions Steering Committee. Public health in America. Bethesda (MD): US Department of Health and Human Services; 1995. Available at: URL: http://www.health.gov/phfunctions/public.htm

5. Institute of Medicine Committee for the Study of the Future of Public Health. The future of public health. Washington DC: National Academy Press; 1988.

6. Ten great public health achievements-United States, 1900-1999. MMWR Morb Mortal Wkly Rep 1999; 48:241-3.

7. Dannenberg AL, Quinlisk MP, Alkon E, Bera N, Cieslak PR, Davis JP, et al. U.S. medical students' rotations in epidemiology and public health at state and local health departments. Acad Med 2002;77: 799-809.

8. Zweiflier J, Evans R. Development of a residency/ MPH program. Fam Med 2001;33:453-8.

9. Dysinger WS. Combining an MPH and/or a preventive medicine residency with family practice training. Fam Med 2002;34:158-9. 